\title{
Existence and Regularity of Solution for Strongly Nonlinear $p(x)$-Elliptic Equation with Measure Data
}

\author{
HASSIB Moulay Cherif ${ }^{1, *}$, AKDIM Youssef ${ }^{1}$, AZROUL Elhoussine $^{2}$ \\ and BARBARA Abdelkrim² \\ ${ }^{1}$ Laboratory LSI, Faculty Polydisciplinary of Taza. University Sidi Mohamed Ben \\ Abdellah, P. O. Box 1223 Taza Gare, Marocco. \\ ${ }^{2}$ Laboratory, LAMA, Faculty of Science dhar El Mahraz Fez. University Sidi \\ Mohamed Ben Abdellah, P. O. Box 1796 Atlas Fez, Morocco.
}

Received 27 March 2016; Accepted 24 October 2016

\begin{abstract}
The first part of this paper is devoted to study the existence of solution for nonlinear $p(x)$ elliptic problem $A(u)=\mu$ in $\Omega, u=0$ on $\partial \Omega$, with a right-hand side measure, where $\Omega$ is a bounded open set of $\mathbb{R}^{N}, N \geqslant 2$ and $A(u)=-\operatorname{div}(a(x, u, \nabla u))$ is a Leray-Lions operator defined from $W_{0}^{1, p(x)}(\Omega)$ in to its dual $W^{-1, p^{\prime}(x)}(\Omega)$. However the second part concerns the existence solution, of the following setting nonlinear elliptic problems $A(u)+g(x, u, \nabla u)=\mu$ in $\Omega, u=0$ on $\partial \Omega$. We will give some regularity results for these solutions.
\end{abstract}

AMS Subject Classifications: 35J20, 35J25, 35J60

Chinese Library Classifications: O175.29

Key Words: Sobolev spaces with variable exponents; strongly nonlinear $p(x)$-elliptic equations with measure data; regularity.

\section{Introduction}

Let $\Omega$ be a bounded open set of $\mathbb{R}^{N},(N \geqslant 2)$. In this paper, we deal with the following Direchlet problem:

$$
\begin{cases}A u+g(x, u, \nabla u)=\mu, & \text { in } \Omega, \\ u=0, & \text { on } \partial \Omega,\end{cases}
$$

with non-standard p-structure which involves a variable growth exponent $p(\cdot)$.

*Corresponding author. Email addresses: cherif_hassib@yahoo.fr (M. C. Hassib), akdimyoussef@yahoo.fr (Y. Akdim), azroulelhoussine@gmail.com (E. Azroul), abdelkrimbarbara@gmail.com (A. Barbara) 
The principal part of the above equation is the operator $A$ defined by

$$
A u=-\operatorname{div}(a(x, u, \nabla u)),
$$

which satisfies the classical Leray-Lions conditions with some variable exponent $p(\cdot)$. However the non linearity $g$ is supposed to satisfy the sign condition and some natural growth with respect to $\nabla u$, but no growth with $u$ is supposed. The second member $\mu$ considered in this paper is not regular that is a Radon measure.

The study of partial differential equation involving $p(x)$ growth conditions has received specific attention in recent decades. This is a consequence of the fact that such equations can be used to model phenomena which arise in mathematical physics. Electro rheological fluids and elastic mechanics are two examples of physical fields which benefit from such kinds of studies.

Problem $(\mathcal{P})$ is studied in [1] where $a=a(x, \nabla u)$ which satisfying the classical LerayLions conditions with some constant exponent $p$ such that: $2-\frac{1}{N}<p<N$. The solution obtained in [1] admits the following regularity $u \in W_{0}^{1, q}(\Omega)$ for all:

$$
1 \leqslant q<\frac{N(p-1)}{N-1}
$$

The approach used by the authors in [1] is to approximate the measure $\mu$ by a sequence $\left(f_{n}\right)$ in $W^{-1, p^{\prime}(x)} \cap L^{1}(\Omega)$ which converge to $\mu$. The limiting process hinges of the proof of the almost pointwise convergence of the sequence $\left(\nabla u_{n}\right)$, where $u_{n}$ is the weak solution of the problem $(\mathcal{P})$ with $\mu=f_{n}$.

When trying to relax the coefficients of $a$, that is $a(\cdot)$ have not a polynomial growths, then the problem $(\mathcal{P})$ is formulated is the general setting of Orlicz spaces generated by an $\mathrm{N}$-function $M$ which appears in the non classical growths of $a(\cdot)$. In this case, we found the work [2] which treat the study of a problem $(\mathcal{P})$ in Orlicz-Sobolev spaces. The solutions obtained in [2] and [3] belongs to $T_{0}^{1, M}(\Omega) \cap W_{0}^{1} L_{B}(\Omega)$ for any $B \in P_{M}$, where $B \in P_{M}$ is a special class of $\mathrm{N}$-functions. For others works, we refer the reader [4-8] and [9].

In the recent years, variable exponent Sobolev spaces have attracted an increasing amount attention, the impulse, for this mainly comes from there physical applications, such in image processing (underline the borders, eliminate the noise) and electro-rheological fluids.

In the framework of variable exponent Sobolev spaces, we list the works $[6,7,10,11]$ and others, where the second member $\mu$ of the problem $(\mathcal{P})$ is taking as an element of $L^{1}(\Omega)$ or is a measure which admits the composition $\mu=f-\operatorname{div}(F)$, with $f \in L^{1}(\Omega)$ and $F \in \prod L^{p(x)}(\Omega)$.

Our purpose in this paper is to study the existence of solution of the problem $(\mathcal{P})$, in the case where the datum $\mu$ is a finite Radon measure and in the case of variable exponent, 
(that is $A$ is a Leray-Lions operator acting from $W_{0}^{1, p(x)}(\Omega)$ in to $W^{-1, p^{\prime}(x)}(\Omega)$ ), with the regularity $u \in W_{0}^{1, q}(\Omega)$ for all: $1 \leqslant q^{-} \leqslant q^{+}<(N /(N-1))\left(p^{-}-1\right)$.

This paper can be seen as a generalization to the variable exponent of the works [1] and as a continuation of the works [6] $\left(f \in L^{1}(\Omega), f \in W^{-1, p^{\prime}(x)}, p=p(x)\right)$.

The paper is organized as follows: In the Section 1, we recall some important definitions and some results of variable exponent Lebesgue and Sobolev spaces. In the Section 2 we give and proof ours mains results.

\section{Framework of the spaces}

In this paragraph, we recall some definition and basic results about the Sobolev spaces with variable exponent.

Let $\Omega$ be a bounded open subset of $I R^{N},(N \geq 2)$, we denote

$$
C_{+}(\bar{\Omega})=\left\{\text { continuous } p(\cdot): \bar{\Omega} \longrightarrow I R \quad \text { such that } 1<p_{-} \leqslant p(x) \leqslant p_{+} \leqslant N\right\},
$$

where

$$
p_{-}=\min \{p(x) / x \in \bar{\Omega}\} \quad \text { and } \quad p_{+}=\max \{p(x) / x \in \bar{\Omega}\} .
$$

We define the variable exponent Lebesgue space for $p(\cdot) \in C_{+}(\bar{\Omega})$ by

$$
L^{p(x)}(\Omega)=\left\{u: \Omega \rightarrow I R \quad \text { measurable } / \int_{\Omega}|u(x)|^{p(x)} \mathrm{d} x<\infty\right\} .
$$

The space $L^{p(x)}(\Omega)$ under the norm

$$
\|u\|_{p(x)}=\inf \left\{\lambda>0, \quad \int_{\Omega}\left|\frac{u(x)}{\lambda}\right|^{p(x)} \mathrm{d} x \leq 1\right\},
$$

is a uniformly convex Banach space, and therefore reflexive. We denote by $L^{p^{\prime}(x)}(\Omega)$ the conjugate space of $L^{p(x)}(\Omega)$ where $\frac{1}{p(x)}+\frac{1}{p^{\prime}(x)}=1$ (see $\left.[12,13]\right)$.

Proposition 2.1. ([12,13]) (Generalized Hölder inequality)

(i) For any $u \in L^{p(x)}(\Omega)$ and $v \in L^{p^{\prime}(x)}(\Omega)$, we have

$$
\left|\int_{\Omega} u v \mathrm{~d} x\right| \leq\left(\frac{1}{p_{-}}+\frac{1}{p_{-}^{\prime}}\right)\|u\|_{p(x)}\|v\|_{p^{\prime}(x)} .
$$

(ii) For all $p_{1}(\cdot), p_{2}(\cdot) \in C_{+}(\bar{\Omega})$ such that $p_{1}(x) \leq p_{2}(x)$, we have $L^{p_{2}(x)}(\Omega) \hookrightarrow L^{p_{1}(x)}(\Omega)$ and the embedding is continuous. 
Proposition 2.2. ([12,13]). If we denote

$$
\rho(u)=\int_{\Omega}|u|^{p(x)} \mathrm{d} x, \quad \forall u \in L^{p(x)}(\Omega),
$$

then, the following assertions holds

(i) $\|u\|_{p(x)}<1 \quad(\operatorname{res} p,=1,>1) \Longleftrightarrow \rho(u)<1 \quad(\operatorname{res} p,=1,>1)$,

(ii) $\|u\|_{p(x)}>1 \Rightarrow\|u\|_{p(x)}^{p_{-}} \leq \rho(u) \leq\|u\|_{p(x)}^{p_{+}}$，

$\|u\|_{p(x)}<1 \Rightarrow\|u\|_{p(x)}^{p_{+}} \leq \rho(u) \leq\|u\|_{p(x)^{\prime}}^{p_{-}}$

(iii) $\|u\|_{p(x)} \rightarrow 0 \Leftrightarrow \rho(u) \rightarrow 0$ and $\|u\|_{p(x)} \rightarrow \infty \Leftrightarrow \rho(u) \rightarrow \infty$.

Now, we define the variable exponent Sobolev space by

$$
W^{1, p(x)}(\Omega)=\left\{u \in L^{p(x)}(\Omega) \quad \text { and } \quad|\nabla u| \in L^{p(x)}(\Omega)\right\},
$$

normed by

$$
\|u\|_{1, p(x)}=\|u\|_{p(x)}+\|\nabla u\|_{p(x)}, \quad \forall u \in W^{1, p(x)}(\Omega) .
$$

We denote by $W_{0}^{1, p(x)}(\Omega)$ the closure of $C_{0}^{\infty}(\Omega)$ in $W^{1, p(x)}(\Omega)$ and we define the Sobolev exponent by $p^{*}(x)=\frac{N p(x)}{N-p(x)}$ for $p(x)<N$.

Proposition 2.3. $([12,14])$

(i) Assuming $1<p_{-} \leq p_{+}<\infty$, the spaces $W^{1, p(x)}(\Omega)$ and $W_{0}^{1, p(x)}(\Omega)$ are separable and reflexive Banach spaces.

(ii) If $q(\cdot) \in C_{+}(\bar{\Omega})$ and $q(x)<p^{*}(x)$ for any $x \in \Omega$, then the embedding $W_{0}^{1, p(x)}(\Omega) \hookrightarrow \hookrightarrow$ $L^{q(x)}(\Omega)$ is continuous and compact.

(iii) Poincaré inequality: there exists a constant $C>0$, such that

$$
\|u\|_{p(x)} \leq C\|\nabla u\|_{p(x)}, \quad \forall u \in W_{0}^{1, p(x)}(\Omega) .
$$

(iv) Sobolev inequality: there exists an other constant $C>0$, such that

$$
\|u\|_{p *(x)} \leq C\|\nabla u\|_{p(x),}, \quad \forall u \in W_{0}^{1, p(x)}(\Omega) .
$$

Remark 2.1. By (iii) of the Proposition 2.3, we deduce that $\|\nabla u\|_{p(x)}$ and $\|u\|_{1, p(x)}$ are equivalent norms in $W_{0}^{1, p(x)}(\Omega)$. 
Definition 2.1. For all $k>0$ and $s \in I R$, the truncation function $T_{k}(\cdot)$ can be defined by

$$
T_{k}(s)=\left\{\begin{array}{lll}
s & \text { if } & |s| \leq k, \\
k \frac{s}{|s|} & \text { if } & |s|>k,
\end{array}\right.
$$

and we define

$$
T_{0}^{1, p(x)}(\Omega):=\left\{u \text { measurable such that } T_{k}(u) \in W_{0}^{1, p(x)}(\Omega), \quad \forall k>0\right\} .
$$

Lemma 2.1. ([15]) Let $g \in L^{r(x)}(\Omega)$ and $g_{n} \in L^{r(x)}(\Omega)$ with $\left\|g_{n}\right\|_{r(x)} \leq C$ for $1<r(x)<\infty$. If $g_{n}(x) \rightarrow g(x)$ a.e. on $\Omega$, then $g_{n} \rightarrow g$ weakly in $L^{r(x)}(\Omega)$.

Lemma 2.2. ([15]) Let $u \in W_{0}^{1, p(x)}(\Omega)$, then $T_{k}(u) \in W_{0}^{1, p(x)}(\Omega)$ for all $k>0$. Moreover, we have

$$
T_{k}(u) \rightarrow u \quad \text { in } W_{0}^{1, p(x)}(\Omega) \quad \text { as } k \rightarrow \infty .
$$

Lemma 2.3. ([10]) Let $p(\cdot)$ be a continuous function in $C_{+}(\bar{\Omega})$ and $u$ a function in $W_{0}^{1, p(x)}(\Omega)$. Suppose $2-\frac{1}{N}<p^{-} \leqslant p^{+} \leqslant N$, and that there exists a constant $C_{1}$ such that

$$
\int_{\{k \leqslant|u| \leqslant k+1\}}|\nabla u|^{p(x)} \mathrm{d} x \leqslant C_{1}, \quad \forall k>0 .
$$

Then, there exists a constant $C_{2}$, depending on $C_{1}$, such that

$$
\|u\|_{W_{0}^{1, q(x)}(\Omega)} \leqslant C_{2}
$$

for all continuous functions $q($.$) on \bar{\Omega}$ satisfying

$$
1 \leqslant q(x)<\frac{N}{N-1}(p(x)-1), \quad \text { for all } x \in \bar{\Omega} .
$$

\section{Main result}

\subsection{Preliminary lemma}

Lemma 3.1. Let $\left(v_{n}\right)_{n}$ be a sequence of functions in $W_{0}^{1, p(x)}(\Omega)$. Suppose that there exists a constant $C>0$ such that, for all $k>0$ :

$$
\int_{\Omega}\left|\nabla \mathrm{T}_{k}\left(v_{n}\right)\right|^{p(x)} \mathrm{d} x \leq C k .
$$

Then, there exists a subsequence still denoted by $\left(v_{n}\right)_{n}$ and a function $v$, such that

$$
\begin{aligned}
& v_{n}(x) \longrightarrow v \text { a.e. in } \Omega, \quad T_{k} v_{n} \rightarrow T_{k} v \text { weakly in } W_{0}^{1, p(\cdot)}(\Omega), \\
& T_{k} v_{n} \longrightarrow T_{k} \text { v strongly in } L^{p(\cdot)}(\Omega) .
\end{aligned}
$$


Proof. We have

$$
\left\|\nabla T_{k}\left(v_{n}\right)\right\|_{p(x)}^{\gamma} \leqslant C k,
$$

where

$$
\gamma=\left\{\begin{array}{lll}
P^{-}, & \text {if } & \left\|\nabla T_{k}\left(v_{n}\right)\right\|_{p(x)}>1 \\
p^{+}, & \text {if } & \left\|\nabla T_{k}\left(v_{n}\right)\right\|_{p(x)} \leqslant 1
\end{array}\right.
$$

Thus

$$
\left\|\nabla T_{k}\left(v_{n}\right)\right\|_{p(x)} \leqslant C_{1} k^{\frac{1}{\gamma}}
$$

where $C_{1}$ is a constant which does not depend on $k$.

From (3.1), it easily follows that $\left(v_{n}\right)_{n}$ is Cauchy sequence in measure. Indeed, we have

$$
k \text { meas }\left\{\left|v_{n}\right|>k\right\}=\int_{\left\{\left|v_{n}\right|>k\right\}}\left|T_{k}\left(v_{n}\right)\right| \mathrm{d} x \leqslant \int_{\Omega}\left|T_{k}\left(v_{n}\right)\right| \mathrm{d} x .
$$

By Hölder inequality, we obtain

$$
k \text { meas }\left\{\left|v_{n}\right|>k\right\} \leqslant\left(\frac{1}{p_{-}}+\frac{1}{p_{-}^{\prime}}\right)\left\|1||_{p^{\prime}(x)}|| T_{k}\left(v_{n}\right)\right\|_{p(x)} .
$$

Therefore

$$
k \text { meas }\left\{\left|v_{n}\right|>k\right\} \leqslant\left(\frac{1}{p_{-}}+\frac{1}{p_{-}^{\prime}}\right)(\operatorname{meas}(\Omega)+1)^{\frac{1}{p_{-}^{\prime}}}|| T_{k}\left(v_{n}\right) \|_{p(x)} .
$$

By the Poincaré inequality we have

$$
\text { meas }\left\{\left|v_{n}\right|>k\right\} \leqslant C_{2} k^{\frac{1}{\gamma}-1} \rightarrow 0, \quad \text { as } k \rightarrow+\infty .
$$

For all $n$ and $m$ in $\mathbb{N}$, for all $\varepsilon>0$ and for every $k>0$, we get

$$
\begin{aligned}
\left\{\left|v_{n}-v_{m}\right| \geqslant \varepsilon\right\}= & \left(\left\{\left|v_{n}-v_{m}\right| \geqslant \varepsilon,\left|v_{n}\right|>k\right\}\right) \cup\left(\left\{\left|v_{n}-v_{m}\right| \geqslant \varepsilon,\left|v_{n}\right| \leqslant k\right\}\right) \\
= & \left(\left\{\left|v_{n}-v_{m}\right| \geqslant \varepsilon,\left|v_{n}\right|>k\right\}\right) \cup\left(\left\{\left|v_{n}-v_{m}\right| \geqslant \varepsilon,\left|v_{n}\right| \leqslant k,\left|v_{m}\right| \leqslant k\right\}\right) \\
& \cup\left(\left\{\left|v_{n}-v_{m}\right| \geqslant \varepsilon,\left|v_{n}\right| \leqslant k,\left|v_{m}\right|>k\right\}\right) .
\end{aligned}
$$

Thus

$$
\left\{\left|v_{n}-v_{m}\right| \geqslant \varepsilon\right\} \subset\left\{\left|v_{n}\right|>k\right\} \cup\left\{\left|T_{k}\left(v_{n}\right)-T_{k}\left(v_{m}\right)\right| \geqslant \varepsilon\right\} \cup\left\{\left|v_{m}\right|>k\right\} .
$$

Therefore

$$
\begin{gathered}
\operatorname{meas}\left(\left\{\left|v_{n}-v_{m}\right| \geqslant \varepsilon\right\}\right) \leqslant \operatorname{meas}\left(\left\{\left|v_{n}\right|>k\right\}\right)+\operatorname{meas}\left(\left\{\left|T_{k}\left(v_{n}\right)-T_{k}\left(v_{m}\right)\right| \geqslant \varepsilon\right\}\right) \\
+\operatorname{meas}\left(\left\{\left|v_{m}\right|>k\right\}\right) .
\end{gathered}
$$

By (3.2), we have for all $\delta>0$ there exists $k_{0}$ such that

$$
\operatorname{meas}\left(\left\{\left|v_{n}\right|>k\right\}\right)<\frac{\delta}{3}, \quad \operatorname{meas}\left(\left\{\left|v_{m}\right|>k\right\}\right)<\frac{\delta}{3}, \quad \forall k \geqslant k_{0}(\delta) .
$$


By (3.1), we have $\left(T_{k}\left(v_{n}\right)\right)_{n}$ is bounded in $W_{0}^{1, p(\cdot)}(\Omega)$, then there exists a subsequence still denoted $\left(T_{k}\left(v_{n}\right)\right)_{n}$ which is strongly compact in $L^{p(x)}(\Omega)$. This means, in particular, that the sequence $\left(T_{k}\left(v_{n}\right)\right)_{n}$ is Cauchy in measure. We then choose $n$ and $m$, such that

$$
\operatorname{meas}\left(\left\{\left|T_{k}\left(v_{n}\right)-T_{k}\left(v_{m}\right)\right| \geqslant \varepsilon\right\}\right)<\frac{\delta}{3} \text {. }
$$

Therefore the sequence $\left(v_{n}\right)_{n}$ is Cauchy in measure, we thus have (up to subsequence, still denoted by $\left.\left(v_{n}\right)_{n}\right)$ which converge almost everywhere in $\Omega$, to some function $v$.

On the other, we have, $T_{k}\left(v_{n}\right) \rightarrow T_{k}(v)$ a.e. in $\Omega$, by combining (3.1) and (Lemma 2.1), we have

and therefore

$$
T_{k} v_{n} \rightarrow T_{k} v \text { weakly in } W_{0}^{1, p(\cdot)}(\Omega) ;
$$

$$
T_{k} v_{n} \rightarrow T_{k} v \text { strongly in } L^{p(\cdot)}(\Omega)
$$

\subsection{Nonlinear elliptic problem: $-\operatorname{div}(a(x, u, \nabla u))=\mu$}

Let $\Omega$ be a bounded open subset of $I^{N}(N \geq 2)$, and let $p \in C_{+}(\bar{\Omega})$, such that

$$
2-\frac{1}{N}<p^{-} \leqslant p^{+} \leqslant N
$$

We consider a Leray-Lions operator $A$ from $W_{0}^{1, p(x)}(\Omega)$ into its dual $W^{-1, p^{\prime}(x)}(\Omega)$, defined by

$$
A u=-\operatorname{div}(a(x, u, \nabla u))
$$

where $a: \Omega \times I R \times I R^{N} \rightarrow I R^{N}$ is a Carathéodory function, satisfying the following conditions

$$
\begin{aligned}
& |a(x, s, \xi)| \leq \beta\left(K(x)+|s|^{p(x)-1}+|\xi|^{p(x)-1}\right), \\
& a(x, s, \xi) \xi \geq \alpha|\xi|^{p(x)}, \\
& {[a(x, s, \bar{\xi})-a(x, s, \bar{\xi})](\xi-\bar{\xi})>0, \text { for all } \xi \neq \bar{\xi} \text { in } I R^{N},}
\end{aligned}
$$

for almost every $x$ in $\Omega$, for every $(s, \xi)$ in $I R \times I R^{N}$, where $K(x)$ is a positive function lying in $L^{p^{\prime}(x)}(\Omega)$ and $\beta>0, \alpha>0$. Let

$$
B_{p(\cdot)}=\left\{q \in \mathcal{C}_{+}(\bar{\Omega}): 1 \leqslant q(x)<\frac{N}{N-1}\left(p^{-}-1\right)\right\} .
$$

Let $\mu \in M_{b}(\Omega), M_{b}(\Omega)$ denotes the set of bounded measures on $\Omega$ (finite Radon measures). Consider the nonlinear elliptic problem:

$$
(\mathcal{P}) \quad \begin{cases}-\operatorname{div}(a(x, u, \nabla u))=\mu & \text { in } \Omega, \\ u=0 & \text { on } \partial \Omega .\end{cases}
$$


Definition 3.1. A measurable function $u$ will be called a weak solution of the problem $(\mathcal{P})$, if

$$
\begin{aligned}
& u \in T_{0}^{1, p(\cdot)}(\Omega) \cap W_{0}^{1, q(\cdot)}, \quad \forall q(\cdot) \in B_{p(\cdot),} \\
& \int_{\Omega} a(x, u, \nabla u) \nabla \varphi \mathrm{d} x=<\mu, \varphi>, \quad \forall \varphi \in D(\Omega) .
\end{aligned}
$$

\subsubsection{Approximate problem}

Consider the approximate equation

$$
\left(\mathcal{P}_{n}\right) \quad\left\{\begin{array}{l}
-\operatorname{div}\left(a\left(x, u_{n}, \nabla u_{n}\right)\right)=f_{n} \quad \text { in } \quad \Omega, \\
u_{n} \in W_{0}^{1, p(\cdot)}(\Omega),
\end{array}\right.
$$

where $\left(f_{n}\right)$ is a smooth function which converges to $\mu$ in the distributional sense such that,

$$
\left\|f_{n}\right\|_{L^{1}(\Omega)} \leqslant\|\mu\|_{M_{b}(\Omega)}
$$

Lemma 3.2. The problem $\left(\mathcal{P}_{n}\right)$ has at last one weak solution $u_{n}$ and there exists a sub-sequence denoted again $\left(u_{n}\right)$ and a function $u$ such that

$$
u_{n} \rightarrow u \text { a.e in } \Omega \text { and } \nabla u_{n} \rightarrow \nabla u \text { a.e. in } \Omega \text {. }
$$

Proof. By [6], the problem $\left(\mathcal{P}_{n}\right)$ has at last one weak solution $u_{n}$.

For $k>0$, by taking $T_{k}\left(u_{n}\right)$ as a test function in (3.11), we deduce that

$$
(\exists C>0): \int_{\Omega} a\left(x, T_{k}\left(u_{n}\right), \nabla T_{k}\left(u_{n}\right)\right) \nabla T_{k}\left(u_{n}\right) \mathrm{d} x \leq C k .
$$

In view of (3.8), we get

$$
\int_{\Omega}\left|\nabla T_{k}\left(u_{n}\right)\right|^{p(x)} \mathrm{d} x \leq C k
$$

By Lemma 3.1 there exists a subsequence still denoted by $u_{n}$ and $u$ such that:

$$
\begin{aligned}
& u_{n} \rightarrow u \text { a.e. in } \Omega ; \quad T_{k}\left(u_{n}\right) \rightarrow T_{k}(u) \text { weakly in } W_{0}^{1, p(\cdot)}(\Omega) ; \\
& T_{k}\left(u_{n}\right) \rightarrow T_{k}(u) \text { strongly in } L^{p(\cdot)}(\Omega) .
\end{aligned}
$$

We can write

$$
\begin{gathered}
0 \leqslant \int_{\Omega}\left(a\left(x, T_{k}\left(u_{n}\right), \nabla T_{k}\left(u_{n}\right)\right)-a\left(x, T_{k}\left(u_{n}\right), \nabla T_{k}(u)\right)\right)\left[\nabla T_{k}\left(u_{n}\right)-\nabla T_{k}(u)\right] \mathrm{d} x \\
=\int_{\Omega}\left(a\left(x, T_{k}\left(u_{n}\right), \nabla T_{k}\left(u_{n}\right)\right)\right)\left[\nabla T_{k}\left(u_{n}\right)-\nabla T_{k}(u)\right] \mathrm{d} x \\
\quad-\int_{\Omega}\left(a\left(x, T_{k}\left(u_{n}\right), \nabla T_{k}(u)\right)\right)\left[\nabla T_{k}\left(u_{n}\right)-\nabla T_{k}(u)\right] \mathrm{d} x .
\end{gathered}
$$


We have

$$
a\left(x, T_{k}\left(u_{n}\right), \nabla T_{k}(u)\right) \rightarrow a\left(x, T_{k}(u), \nabla T_{k}(u)\right) \text { a.e. in } \Omega .
$$

By combining (3.7), (3.16) and the dominated convergence theorem we get

$$
a\left(x, T_{k}\left(u_{n}\right), \nabla T_{k}(u)\right) \rightarrow a\left(x, T_{k}(u), \nabla T_{k}(u)\right) \text { stongly in }\left(L^{P^{\prime}(x)}(\Omega)\right)^{N} .
$$

Therefore

$$
\int_{\Omega}\left(a\left(x, T_{k}\left(u_{n}\right), \nabla T_{k}(u)\right)\right)\left[\nabla T_{k}\left(u_{n}\right)-\nabla T_{k}(u)\right] \mathrm{d} x \rightarrow 0, \quad \text { as } n \rightarrow \infty .
$$

Let $\eta>0$ and taking $T_{\eta}\left(u_{n}-T_{k}(u)\right)$ as test function in (3.11), we obtain

$$
\int_{\Omega} a\left(x, u_{n}, \nabla u_{n}\right) \nabla T_{\eta}\left(u_{n}-T_{k}(u)\right) \mathrm{d} x \leq C \eta .
$$

For the sake of simplicity, we write only $\varepsilon(n, \eta)$ to mean all quantities (possibly different) such that

$$
\lim _{n \rightarrow \infty} \lim _{\eta \rightarrow 0} \varepsilon(n, \eta)=0
$$

On the other hand

$$
\begin{aligned}
& \quad \int_{\Omega} a\left(x, u_{n}, \nabla u_{n}\right) \nabla T_{\eta}\left(u_{n}-T_{k}(u)\right) \mathrm{d} x \\
& =\int_{\left\{\left|u_{n}-T_{k}(u)\right| \leqslant \eta\right\} \cap\left\{\left|u_{n}\right| \leqslant k\right\}} a\left(x, T_{k}\left(u_{n}\right), \nabla T_{k}\left(u_{n}\right)\right)\left(\nabla T_{k}\left(u_{n}\right)-\nabla T_{k}(u)\right) \mathrm{d} x \\
& \quad+\int_{\left\{\left|u_{n}-T_{k}(u)\right| \leqslant \eta\right\} \cap\left\{\left|u_{n}\right|>k\right\}} a\left(x, u_{n}, \nabla u_{n}\right)\left(\nabla u_{n}-\nabla T_{k}(u)\right) \mathrm{d} x \\
& =\int_{\left\{\left|T_{k}\left(u_{n}\right)-T_{k}(u)\right| \leqslant \eta\right\} \cap\left\{\left|u_{n}\right| \leqslant k\right\}} a\left(x, T_{k}\left(u_{n}\right), \nabla T_{k}\left(u_{n}\right)\right)\left(\nabla T_{k}\left(u_{n}\right)-\nabla T_{k}(u)\right) \mathrm{d} x \\
& \quad+\int_{\left\{\left|u_{n}-T_{k}(u)\right| \leqslant \eta\right\} \cap\left\{\left|u_{n}\right|>k\right\}} a\left(x, u_{n}, \nabla u_{n}\right) \nabla u_{n} \mathrm{~d} x \\
& \quad-\int_{\left\{\left|u_{n}-T_{k}(u)\right| \leqslant \eta\right\} \cap\left\{\left|u_{n}\right|>k\right\}} a\left(x, u_{n}, \nabla u_{n}\right) \nabla T_{k}(u) \mathrm{d} x .
\end{aligned}
$$

By (3.8) the second term of the right-hand side of (3.17) satisfies

$$
\int_{\left\{\left|u_{n}-T_{k}(u)\right| \leqslant \eta\right\} \cap\left\{\left|u_{n}\right|>k\right\}} a\left(x, u_{n}, \nabla u_{n}\right) \nabla u_{n} \mathrm{~d} x \geqslant 0,
$$

the third term of the right side of (3.17) satisfies

$$
\begin{aligned}
& \int_{\left\{\left|u_{n}-T_{k}(u)\right| \leqslant \eta\right\} \cap\left\{\left|u_{n}\right|>k\right\}} a\left(x, u_{n}, \nabla u_{n}\right) \nabla T_{k}(u) \mathrm{d} x \\
= & \int_{\left\{\left|u_{n}-T_{k}(u)\right| \leqslant \eta\right\} \cap\left\{\left|u_{n}\right|>k\right\}} a\left(x, T_{k+\eta}\left(u_{n}\right), \nabla T_{k+\eta}\left(u_{n}\right)\right) \nabla T_{k}(u) \mathrm{d} x .
\end{aligned}
$$


Since $a\left(x, T_{k+\eta}\left(u_{n}\right), \nabla T_{k+\eta}\left(u_{n}\right)\right)$ is bounded in $\left(L^{p^{\prime}(x)}(\Omega)\right)^{N}$, there exists $h_{\eta+k} \in\left(L^{p^{\prime}(x)}(\Omega)\right)^{N}$ such that

$$
a\left(x, T_{k+\eta}\left(u_{n}\right), \nabla T_{k+\eta}\left(u_{n}\right)\right) \rightarrow h_{\eta+k}, \text { weakly in }\left(L^{p^{\prime}(x)}(\Omega)\right)^{N} .
$$

Since,

$$
\nabla T_{k}(u) \chi_{\left\{\left|u_{n}-T_{k}(u)\right| \leqslant \eta\right\} \cap\left\{\left|u_{n}\right|>k\right\}} \rightarrow 0 \text { strongly in }\left(L^{p(x)}(\Omega)\right)^{N},
$$

as $n \rightarrow \infty$ and $\eta \rightarrow 0$. Thus

$$
\begin{aligned}
& \int_{\left\{\left|T_{k}\left(u_{n}\right)-T_{k}(u)\right| \leqslant \eta\right\} \cap\left\{\left|u_{n}\right| \leqslant k\right\}} a\left(x, T_{k}\left(u_{n}\right), \nabla T_{k}\left(u_{n}\right)\right)\left(\nabla T_{k}\left(u_{n}\right)-\nabla T_{k}(u)\right) \mathrm{d} x \\
& \leqslant C \eta+\varepsilon(n, \eta) .
\end{aligned}
$$

On the other hand,

$$
\begin{aligned}
& \int_{\left\{\left|T_{k}\left(u_{n}\right)-T_{k}(u)\right| \leqslant \eta\right\} \cap\left\{\left|u_{n}\right|>k\right\}} a\left(x, T_{k}\left(u_{n}\right), \nabla T_{k}\left(u_{n}\right)\right)\left(\nabla T_{k}\left(u_{n}\right)-\nabla T_{k}(u)\right) \mathrm{d} x \\
\leqslant & \int_{\left\{\left|T_{k}\left(u_{n}\right)-T_{k}(u)\right| \leqslant \eta\right\} \cap\left\{\left|u_{n}\right|>k\right\}}\left|a\left(x, T_{k}\left(u_{n}\right), \nabla T_{k}\left(u_{n}\right)\right)\right|\left|\nabla T_{k}(u)\right| \mathrm{d} x .
\end{aligned}
$$

We have

$$
\begin{aligned}
& \left|\nabla T_{k}(u)\right| \chi_{\left\{\left|T_{k}\left(u_{n}\right)-T_{k}(u)\right| \leqslant \eta\right\} \cap\left\{\left|u_{n}\right|>k\right\}} \rightarrow 0, \text { a.e. in } \Omega \text { as } n \rightarrow \infty, \\
& \left|T_{k}(u)\right| \chi_{\left\{\left|T_{k}\left(u_{n}\right)-T_{k}(u)\right| \leqslant \eta\right\} \cap\left\{\left|u_{n}\right|>k\right\}} \leqslant\left|T_{k}(u)\right|,
\end{aligned}
$$

thus by Lebesgue dominated convergence theorem, we deduce that

$$
\left|\nabla T_{k}(u)\right| \chi_{\left\{\left|T_{k}\left(u_{n}\right)-T_{k}(u)\right| \leqslant \eta\right\} \cap\left\{\left|u_{n}\right|>k\right\}} \rightarrow 0, \text { in } L^{p(\cdot)}(\Omega), \quad \text { as } n \rightarrow \infty
$$

Since the sequence $\left(\left|a\left(x, T_{k}\left(u_{n}\right), \nabla T_{k}\left(u_{n}\right)\right)\right|\right)_{n}$ is bonded in $\left(L^{p^{\prime}(\cdot)}(\Omega)\right)^{N}$,

$$
\int_{\left\{\left|T_{k}\left(u_{n}\right)-T_{k}(u)\right| \leqslant \eta\right\} \cap\left\{\left|u_{n}\right|>k\right\}}\left|a\left(x, T_{k}\left(u_{n}\right), \nabla T_{k}\left(u_{n}\right)\right)\right|\left|\nabla T_{k}(u)\right| \mathrm{d} x \rightarrow 0, \quad \text { as } n \rightarrow \infty
$$

Thus

$$
\int_{\left\{\left|T_{k}\left(u_{n}\right)-T_{k}(u)\right| \leqslant \eta\right\} \cap\left\{\left|u_{n}\right|>k\right\}} a\left(x, T_{k}\left(u_{n}\right), \nabla T_{k}\left(u_{n}\right)\right)\left(\nabla T_{k}\left(u_{n}\right)-\nabla T_{k}(u)\right) \mathrm{d} x \leqslant \varepsilon(n) .
$$

On the other hand,

$$
\int_{\left.\left\{\left|T_{k}\left(u_{n}\right)-T_{k}(u)\right|>\eta\right\}\right\}} a\left(x, T_{k}\left(u_{n}\right), \nabla T_{k}\left(u_{n}\right)\right)\left(\nabla T_{k}\left(u_{n}\right)-\nabla T_{k}(u)\right) \mathrm{d} x \rightarrow 0 \text { as } n \rightarrow \infty,
$$

since

$$
\operatorname{meas}\left(\left\{\left|T_{k}\left(u_{n}\right)-T_{k}(u)\right|>\eta\right\}\right) \rightarrow 0 \text { as } n \rightarrow \infty
$$


and by Hölder inequality we have, $a\left(x, T_{k}\left(u_{n}\right), \nabla T_{k}\left(u_{n}\right)\right)\left(\nabla T_{k}\left(u_{n}\right)-\nabla T_{k}(u)\right)$ is bounded in $L^{1}(\Omega)$.

Thus by passing to the limit over $n$ and $\eta$, we get

$$
\lim _{n \longrightarrow \infty} \int_{\Omega}\left(a\left(x, T_{k}\left(u_{n}\right), \nabla T_{k}\left(u_{n}\right)\right)-a\left(x, T_{k}\left(u_{n}\right), \nabla T_{k}(u)\right)\right)\left[\nabla T_{k}\left(u_{n}\right)-\nabla T_{k}(u)\right] \mathrm{d} x=0 .
$$

We conclude by [11, Lemma 4.4$]$ that there exists a sub-sequence denoted again $\left(u_{n}\right)$ such that

$$
\nabla u_{n} \rightarrow \nabla u \text { a.e. in } \Omega .
$$

This completes the proof of the lemma.

\subsubsection{Result of existence}

Theorem 3.1. Assume that (3.5) and (3.7)-(3.9) hold and $\mu \in M_{b}(\Omega)$, then there exists at last one weak solution of the problem $(\mathcal{P})$.

Proof. Let $k>0$, we define the function $\psi_{k}$ as:

$$
\begin{cases}\psi_{k}(x)=1, & \text { if } x>k+1, \\ \psi_{k}(x)=x-k, & \text { if } k \leqslant x \leqslant k+1, \\ \psi_{k}(x)=0, & \text { if }-k \leqslant x \leqslant k, \\ \psi_{k}(x)=x+k, & \text { if }-k-1 \leqslant x \leqslant-k, \\ \psi_{k}(x)=-1, & \text { if } x<-k-1 .\end{cases}
$$

By taking $\psi_{k}\left(u_{n}\right)$ as test function in (3.11) and using (3.8) one has:

$$
\int_{D_{k, n}}\left|\nabla u_{n}\right|^{p(x)} \mathrm{d} x \leqslant \frac{1}{\alpha}\|\mu\|_{M_{b}(\Omega)},
$$

with

$$
D_{k, n}=\left\{x \in \Omega, k \leqslant\left|u_{n}(x)\right| \leqslant k+1\right\} .
$$

In view of Lemma 2.3, there exists a constant $C$ that does not depend on $n$ such that

$$
\left\|u_{n}\right\|_{W_{0}^{1, q(x)}(\Omega)} \leqslant C, \quad \forall q(\cdot) \in B_{p(\cdot)} \cdot
$$

Hence $\left(u_{n}\right)$ is relatively compact in $W_{0}^{1, q(x)} \forall q(\cdot) \in B_{p(\cdot)}$, thus there exists a sub-sequence denoted again by $\left(u_{n}\right)$ such that

$$
u_{n} \rightarrow u \text { weakly in } W_{0}^{1, q(x)}, \quad u_{n} \rightarrow u \text { strongly in } L^{q(x)}, \quad u_{n} \rightarrow u \text { a.e. in } \Omega .
$$

By Lemma 3.2, we conclude that

$$
a\left(x, u_{n}, \nabla u_{n}\right) \rightarrow a(x, u, \nabla u), \quad \text { a.e in } \Omega .
$$


Combining (3.7) and (3.22), yields

$$
\left\|a\left(x, u_{n}, \nabla u_{n}\right)\right\|_{L^{r(x)}} \leqslant C,
$$

for every $1<r(x)<N /(N-1)$. Moreover, using Lemma 2.1, we can write

$$
a\left(x, u_{n}, \nabla u_{n}\right) \rightarrow a(x, u, \nabla u) \text { weakly in } L^{r(x)}(\Omega) .
$$

It is now possible to pass to the limit in (3.11), we conclude that $u$ is the weak solution of the problem $(\mathcal{P})$.

\subsection{Strongly nonlinear elliptic problem: $-\operatorname{div}(a(x, u, \nabla u))+g(x, u, \nabla u)=\mu$}

Consider the equation

$$
\left(\mathcal{P}^{\prime}\right) \quad\left\{\begin{array}{l}
-\operatorname{div}(a(x, u, \nabla u))+g(x, u, \nabla u)=\mu \quad \text { in } \Omega, \\
u=0 \quad \text { on } \partial \Omega,
\end{array}\right.
$$

where $a$ satisfies (3.6)-(3.9), $\mu$ lie in $M(\Omega)$, and the non linear term $g: \Omega \times I R \times I R^{N} \rightarrow I R$, is a Carathédory function satisfying for almost every $x \in \Omega$ and for all $s \in I R, \zeta \in I R^{N}$ the following conditions:

$$
\begin{aligned}
& g(x, s, \xi) . s \geqslant 0 \\
& |g(x, s, \xi)| \leq b(|s|)\left(c(x)+|\xi|^{p(x)}\right)
\end{aligned}
$$

where $b: I R^{+} \rightarrow I R^{+}$is a continuous growth function and $c: \Omega \rightarrow I R^{+}$with $c \in L^{1}(\Omega)$.

Definition 3.2. We say that $u$ is a weak solution of the problem (3.23) if

$$
\begin{gathered}
u \in W_{0}^{1, q(\cdot)}, \quad \forall q(\cdot) \in B_{p(\cdot)}, \quad a(x, u, \nabla u) \in L^{1}(\Omega), \quad g(x, u, \nabla u) \in L^{1}(\Omega), \\
\int_{\Omega} a(x, u, \nabla u) \nabla \varphi \mathrm{d} x+\int_{\Omega} g(x, u, \nabla u) \varphi \mathrm{d} x=<\mu, \varphi>, \quad \forall \varphi \in D(\Omega) .
\end{gathered}
$$

Theorem 3.2. Let a satisfy (3.6)-(3.9), $g$ satisfy (3.24)-(3.25) and $\mu$ lies in $M(\Omega)$. Then there exists a weak solution of (3.23).

Proof. Let $\left(f_{n}\right)$ be a sequence of $L^{1}(\Omega) \cap W^{-1, p^{\prime}(\cdot)}(\Omega)$ which converges to $\mu$ in the distributional sense and such that

$$
\left\|f_{n}\right\|_{L^{1}(\Omega)} \leqslant\|\mu\|_{M_{b}(\Omega)}, \quad \forall n \in \mathbb{N} .
$$

By [6] there exists a weak solution $\left(u_{n}\right)$ of the problem (3.23) with $f_{n}=\mu$ which satisfies:

$$
u_{n} \in W_{0}^{1, p(x)}(\Omega), \quad g\left(x, u_{n}, \nabla u_{n}\right) \in L^{1}(\Omega),
$$




$$
\begin{gathered}
\int_{\Omega} a\left(x, u_{n}, \nabla u_{n}\right) \cdot \nabla v \mathrm{~d} x+\int_{\Omega} g\left(x, u_{n}, \nabla u_{n}\right) v \mathrm{~d} x=\left\langle f_{n}, v>,\right. \\
\forall v \in W_{0}^{1, p(x)}(\Omega) \cap L^{\infty}(\Omega) .
\end{gathered}
$$

Following the lines of [1], it is easy to deduce that

$$
\left\|g\left(x, u_{n}, \nabla u_{n}\right)\right\|_{L^{1}(\Omega)} \leqslant\left\|f_{n}\right\|_{L^{1}(\Omega)} .
$$

Setting $h_{n}=f_{n}-g\left(x, u_{n}, \nabla u_{n}\right)$, by (3.27) and (3.29) we have

$$
\left\|h_{n}\right\|_{L^{1}(\Omega)} \leqslant 2|| \mu \|_{M_{b}(\Omega)} .
$$

Note that $u_{n}$ is the solution of the problem (3.11) with a right-hand side is $h_{n}$. By the Section 2.2 the sequence $\left(u_{n}\right)$ is relatively compact in $W_{0}^{1, q(x)}(\Omega), \forall q(\cdot) \in B_{p(\cdot)}$. Then we can assume (after extraction of a sub-sequence, still denoted by $\left(u_{n}\right)$ )

$$
\begin{aligned}
& u_{n} \rightarrow u \text { weakly in } W_{0}^{1, q(x)}, \text { for all } q(\cdot) \in B_{p(\cdot),} \\
& u_{n} \rightarrow u \text { in } L^{q(x)}, \quad u_{n} \rightarrow u \text { a.e. in } \Omega, \\
& a\left(x, u_{n}, \nabla u_{n}\right) \rightarrow a(x, u, \nabla u) \text { weakly in } L^{r(x)}(\Omega), \quad \text { for } 1<r(x)<N /(N-1) .
\end{aligned}
$$

Now, we prove that

$$
g\left(x, u_{n}, \nabla u_{n}\right) \rightarrow g(x, u, \nabla u) \text { strongly in } L^{1}(\Omega),
$$

we have $g_{n}\left(x, u_{n}, \nabla u_{n}\right) \rightarrow g(x, u, \nabla u)$ a.e. in $\Omega$, using the Vitali convergence theorem, it sufficient to show that $g_{n}\left(x, u_{n}, \nabla u_{n}\right)$ is uniformly equi-integrable. Indeed, let $h>0$, taking $T_{1}\left(u_{n}-T_{h}\left(u_{n}\right)\right)$ as a test function in (3.28), we obtain

$$
\begin{aligned}
& \int_{\Omega} a\left(x, u_{n}, \nabla u_{n}\right) \cdot \nabla T_{1}\left(u_{n}-T_{h}\left(u_{n}\right)\right) \mathrm{d} x+\int_{\Omega} g\left(x, u_{n}, \nabla u_{n}\right) T_{1}\left(u_{n}-T_{h}\left(u_{n}\right)\right) \mathrm{d} x \\
= & \int_{\Omega} f_{n} T_{1}\left(u_{n}-T_{h}\left(u_{n}\right)\right) \mathrm{d} x,
\end{aligned}
$$

it follows that

$$
\int_{\left\{\left|u_{n}\right| \geqslant h\right\}} g\left(x, u_{n}, \nabla u_{n}\right) T_{1}\left(u_{n}-T_{h}\left(u_{n}\right)\right) \mathrm{d} x \leqslant \int_{\left\{\left|u_{n}\right| \geqslant h\right\}} f_{n} T_{1}\left(u_{n}-T_{h}\left(u_{n}\right)\right) \mathrm{d} x .
$$

Then

$$
\begin{aligned}
& \int_{\left\{\left|u_{n}\right| \geqslant h+1\right\}}\left|g\left(x, u_{n}, \nabla u_{n}\right)\right| \mathrm{d} x \\
\leqslant & \int_{\left\{\left|u_{n}\right| \geqslant h\right\}} g\left(x, u_{n}, \nabla u_{n}\right) T_{1}\left(u_{n}-T_{h}\left(u_{n}\right)\right) \mathrm{d} x \\
\leqslant & \int_{\left\{\left|u_{n}\right| \geqslant h\right\}} f_{n} T_{1}\left(u_{n}-T_{h}\left(u_{n}\right)\right) \mathrm{d} x
\end{aligned}
$$




$$
\leqslant\left(\frac{1}{p_{-}}+\frac{1}{p_{-}^{\prime}}\right)\left\|f_{n}\right\|_{-1, p^{\prime}(\cdot)}\left\|T_{1}\left(u_{n}-T_{h}\left(u_{n}\right)\right)\right\|_{1, p(\cdot)} \rightarrow 0, \quad \text { as } h \rightarrow \infty .
$$

Thus, for all $\varepsilon>0$, there exists $h(\varepsilon)>0$ such that

$$
\int_{\left\{\left|u_{n}\right| \geqslant h(\varepsilon)\right\}}\left|g\left(x, u_{n}, \nabla u_{n}\right)\right| \mathrm{d} x \leqslant \frac{\varepsilon}{2} .
$$

On the other hand, for any measurable subset $D \subset \Omega$, we have

$$
\begin{aligned}
& \int_{D}\left|g\left(x, u_{n}, \nabla u_{n}\right)\right| \mathrm{d} x \\
\leqslant & \int_{D} b(h)\left(c(x)+\left|\nabla T_{h}\left(u_{n}\right)\right|^{p(x)}\right) \mathrm{d} x+\int_{\left\{\left|u_{n}\right| \geqslant h(\varepsilon)\right\}}\left|g\left(x, u_{n}, \nabla u_{n}\right)\right| \mathrm{d} x .
\end{aligned}
$$

There exists $\beta(\varepsilon)>0$ such that

$$
b(h) \int_{D}\left(c(x)+\left|\nabla T_{h}\left(u_{n}\right)\right|^{p(x)}\right) \mathrm{d} x \leqslant \frac{\varepsilon}{2}, \quad \text { for meas }(D) \leqslant \beta(\varepsilon) .
$$

Thus

$$
\int_{D}\left|g\left(x, u_{n}, \nabla u_{n}\right)\right| \mathrm{d} x \leqslant \varepsilon, \quad \text { with meas }(D) \leqslant \beta(\varepsilon) .
$$

By Vitali convergence theorem we deduce that $g\left(x, u_{n}, \nabla u_{n}\right) \rightarrow g(x, u, \nabla u)$ strongly in $L^{1}(\Omega)$. Now, taking $\varphi \in D(\Omega)$ as test function in (3.28), we have

$$
\int_{\Omega} a\left(x, u_{n}, \nabla u_{n}\right) \cdot \nabla \varphi \mathrm{d} x+\int_{\Omega} g\left(x, u_{n}, \nabla u_{n}\right) \varphi \mathrm{d} x=<f_{n}, \varphi>.
$$

By letting $n$ tends to $\infty$, we obtain

$$
\int_{\Omega} a(x, u, \nabla u) \nabla \varphi \mathrm{d} x+\int_{\Omega} g(x, u, \nabla u) \varphi \mathrm{d} x=\langle\mu, \varphi>, \quad \forall \varphi \in D(\Omega) .
$$

We have show that

$$
\begin{aligned}
& u \in W_{0}^{1, q(\cdot)}, \forall q(\cdot) \in B_{p(\cdot)} ; \quad g(x, u, \nabla u) \in L^{1}(\Omega), \\
& a(x, u, \nabla u) \in L^{r(x)}(\Omega), \quad \text { for } 1<r(x)<\frac{N}{N-1}, \\
& \int_{\Omega} a(x, u, \nabla u) \nabla \varphi \mathrm{d} x+\int_{\Omega} g(x, u, \nabla u) \varphi \mathrm{d} x=<\mu, \varphi>, \quad \forall \varphi \in D(\Omega),
\end{aligned}
$$

thus $u$ is the weak solution of problem (3.23).

Exemple 3.1. We consider the following functions

$$
a(x, u, \nabla u)=|\nabla u|^{p(x)-2} \nabla u, \quad \text { and } g(x, u, \nabla u)=\left(1+|\nabla u|^{p(x)}\right)|u|^{p(x)-2} u .
$$


It is clear that $a(x, u, \nabla u)$ and $g(x, u, \nabla u)$ verifies (3.6)-(3.9) and (3.24)-(3.25) respectively, then by Theorem 3.2 for all $\mu \in M(\Omega)$ there exists a weak solution of the problem

$$
\begin{cases}-\operatorname{div}\left(|\nabla u|^{p(x)-2} \nabla u\right)+\left(1+|\nabla u|^{p(x)}\right)|u|^{p(x)-2} u=\mu, & \text { in } \Omega, \\ u=0, & \text { on } \partial \Omega .\end{cases}
$$

\section{Acknowledgement}

The authors would like to thank the referees for carefully reading the manuscript and for their useful suggestions.

\section{References}

[1] Boccardo L. and Gallouët T., Nonlinear elliptic and parabolic equations involving measure data. J. Funct. Anal., 87 (1989), 149-169.

[2] Dong GE., Elliptic Equation with measure data in Orlicz spaces. Electronic Journal of Differential equation, (2008), 1-10.

[3] GE Dong and Fan X. L., Existence results for some nonlinear elliptic equations with measure data in Orlicz-Sobolev spaces. Boundary Value Problems, 2015 (18) (2015).

[4] Prignet A., Conditions aux limites non homogènes pour des problèmes elliptiques avec second membre mesure. Ann. Fac. Sciences de Toulouse, 1997 (6) 297C318.

[5] Porreta A., Nonlinear equations with natural growth terms and measure data. Electron. J. Differ. Equ. Conf., 09 (2002), 183-202.

[6] Azroul E., Barbara A. and Hjiaj H., Strongly nonlinear $p(x)$-elliptic problem with $L^{1}$-data. African Diaspora Journal of Mathematics, 16 (2) (2014), 1-22.

[7] Azroul E., Benboubker M. B. and Rhoudaf M., On some $p(x)$-quasilinear problem with righthand side measure. Mathematics and Computers in Simulation, 102 (2014), 117-103.

[8] Boccardo L. and Gallouët T., Nonlinear elliptic equations with right hand side measures. Comm. Partial Differential Equations, 17 (1992), 641-655.

[9] Bénilan P., Boccardo L., Gallouët T., Gariepy R., Pierre M., and Vázquez J. L., An L1-theory of existence and uniqueness of solutions of nonlinear elliptic equations. Ann. Scuola Norm. Sup. Pisa Cl. Sci., 4 (1995), 241-273.

[10] Bendahmane M. B. and Wittbold P., Renormalized solutions for nonlinear elliptic equations with variable exponents and $L^{1}$ data. Nonl. Anal., 70 (2009), 567-583.

[11] Azroul E., Hiiaj H. and Touzani A., Existence and regularity of entropy solutions for strongly nonlinear $p(x)$-elliptic equations. Electronic Journal of Differential Equations, 68 (2013), 1-27.

[12] Fan X. L. and Zhao D., On the generalized Orlicz-Sobolev Space $W^{k, p(x)}(\Omega)$. J. Gansu Educ. College 1, 1 (1998), 1-6.

[13] Zhao D., Qiang W. J. and Fan X. L., On generalized Orlicz spaces $L^{p(x)}(\Omega)$. J. Gansu Sci., 9 (1997), 1-7.

[14] Harjulehto P. and Hästö P., Sobolev inequalities for variable exponents attaining the values 1 and n. Publ. Mat., 52 (2008), 347-363.

[15] Benboubker M. B., Azroul E. and Barbara A., Quasilinear elliptic problems with nonstandard growths. Electronic Journal of Differential Equations, 62 (2011), 1-16. 
[16] Benkirane A. and Elmahi A., An existence for a strongly nonlinear elliptic problem in Orlicz spaces. Nonl. Anal., 36 (1999), 11-24.

[17] Dienning L., Harjulehto P., Hästö P. and Ružička M., Lebesgue and Sobolev Spaces with Variable Exponents. Lecture Notes in Mathematics, Springer, Heidelberg, Germany, 2017.

[18] Sanchón M. and Ubrano J.M., Entropy solutions for the $p(x)$-Laplace equation. Trans. Amer. Math. Soc., 361(12) (2009), 6387-6405.

[19] Yao J., Solutions for Neumann boundary value problems involving $p(x)$-Laplace operators. Nonl. Anal., 68 (2008), 1271-1283. 